\title{
POLÍTICAS DE AMPLIACIÓN DE LA JORNADA ESCOLAR EN RIO GRANDE DO SUL (BRASIL): UNA REFLEXIÓN CURRICULAR
}

\section{Integral education policies in the state of Rio Grande do Sul (Brazil): A Curricular Reflection}

\author{
ROBERTO RAFAEL DIAS DA SILVA*1 \\ http://orcid.org/0000-0001-6927-3435 \\ * Universidade do Vale do Rio dos Sinos. Brasil. \\ Correspondencia: robertoddsilva@yahoo.com.br
}

Recibido: $30-10-2017$

Revisado: 30-12-2017

Aceptado: 28-11-2018

Resumen: En el presente artículo se busca establecer un mapeo de los conceptos de conocimiento escolar emergentes de las políticas de ampliación de la jornada escolar desarrollada en Brasil, en el contexto de Rio Grande do Sul. Las políticas curriculares examinadas focalizan la constitución de "comunidades de aprendizaje". Entienden la ampliación de la jornada como una apuesta en actividades pedagógicas innovadoras, con énfasis en los intereses y las experiencias culturales de los estudiantes. Se diferencian en el modo de organización curricular, dado que uno de los programas se centra en habilidades genéricas, mientras que el otro dispone expectativas de aprendizaje para las diferentes franjas etarias. Se constata una fragilidad de los procesos de selección y organización del conocimiento escolar.

Palabras clave: políticas educacionales; ampliación de la jornada escolar; conocimiento.

\begin{abstract}
In this paper we want to map conceptions of school knowledge emerging in the current policies for increasing the workday in schools in Rio Grande do Sul context. Curricular policies we have examined focus on establishment of 'learning communities'. They take the increasing of the workday in schools as a proposal encouraging creative pedagogical activities stressed on students' interests and cultural experiences. However, they are different in the terms of curricular organization as one of their programs is centered on general skills, while the other shows learning expectations for different age ranges. We have found weak points in the processes of selection and organization of the school knowledge, as curricular policies neglect possibilities of cultural transmission concerning schooling.
\end{abstract}

Keywords: education policies; extension of school hours; knowledge.

1 Doctor en Educación de la Universidade do Vale do Rio dos Sinos (Brasil), magíster en Educación (UNISINOS); licenciado en Pedagogía. Líneas de trabajo: curriculum, políticas educacionales, juventud, educación. 


\section{INTRODUCCIÓN}

Educar es conseguir que el niño sobrepase las fronteras que, tantas veces, le fueron trazadas como destino por el nacimiento, la familia o la sociedad (Nóvoa, 2009, p. 32). ${ }^{2}$

Con el presente texto ${ }^{3}$ se pretende establecer un ejercicio de análisis de las políticas contemporáneas que atribuyen centralidad a la ampliación de la jornada escolar en Brasil. Este abordaje se inscribe en el campo de los Estudios Curriculares, y se le atribuye centralidad analítica a la noción de conocimiento escolar. Para ello, se revisarán dos políticas de implementación en el Estado de Rio Grande do Sul, a saber: el programa Más Educación, desarrollado desde una política inductora del gobierno federal, y el proyecto Escuela de Turno completo, política recientemente creada en el contexto del estado. De forma general, el presente trabajo moviliza sus esfuerzos hacia la producción de un diagnóstico crítico de las concepciones de conocimiento escolar convocadas en el proceso de implementación de las políticas referidas.

En este momento, importa resaltar que, a lo largo del último siglo, la escolarización pública desarrolló significativos avances respecto a sus posibilidades democratizadoras. No obstante, de acuerdo a António Nóvoa (2009), al describir la concepción de escuela, fabricada a lo largo del siglo $\mathrm{XX}$, se hace relevante indicar que la institución referida "se fue desarrollando por acumulación de misiones y contenidos, en una especie de desbordamiento, que la llevó a asumir una infinidad de tareas" (p. 52). En su abordaje, aunque la escuela haya avanzado en la composición de una pauta democratizadora, en muchos aspectos, todavía está consolidada una imagen institucional centrada en la regeneración, la salvación o la reparación de la sociedad (Nóvoa, 2009). El debate educacional del siglo XX, en sus diferentes matices, supone una formación ciudadana, que extrapole los límites de la instrucción. La articulación entre educación y ciudadanía, asociada al contexto de construcción del Estado Moderno, se posiciona como uno de los grandes presupuestos políticos de la Modernidad (Dubet, 2011). Las concepciones educacionales, movilizadas desde este periodo, en su multiplicidad de perspectivas emergentes, desafían el campo pedagógico para "ir más allá del acto de instruir y promover una auténtica educación del carácter y el espíritu" (Nóvoa, 2009). Respecto a esta cuestión, nos indica el historiador, se estructura la Modernidad Pedagógica.

No espanta, por ello, el éxito del concepto de educación integral, sin duda aquel que mejor traduce el proyecto de la modernidad escolar. Al marcar el deseo de alargar el esfuerzo educativo al "conjunto de las actividades del individuo en formación”, [la educación integral] revela la desmedida ambición pedagógica (Nóvoa, 2009, p. 55).

\footnotetext{
${ }^{2}$ Las citas de este artículo fueron traducidas del portugués al castellano por el autor.

${ }^{3}$ Este trabajo recibió apoyo financiero del Consejo Nacional de Desarrollo Científico y Tecnológico (CNPQ).
} 
Retomando el abordaje propuesto por Nóvoa (2009), en una lectura de los dilemas contemporáneos, la escuela de nuestro tiempo se presenta como uno de sus "peligros", una organización

a dos velocidades: por un lado, una escuela concebida esencialmente como un centro de acogimiento social, para los pobres, con una fuerte retórica en la ciudadanía y la participación; por otro lado, una escuela claramente centrada en el aprendizaje y en las tecnologías, destinada a formar a los hijos de los ricos (p. 67).

Dicha posibilidad se aproxima al diagnóstico propuesto por Libâneo (2012) acerca de las políticas de educación movilizadas en Brasil, en las que se evidencia una escuela del conocimiento para los ricos y otra marcada por el acogimiento social para los pobres.

En el límite, Nóvoa propone que la construcción de la escuela contemporánea articule ciudadanía con aprendizaje, de manera que la institución no pierda sus prioridades. Entre las cuestiones emergentes de su análisis, se indica la necesidad de "asegurar que todos los niños adquieran una base común de conocimientos, cualquier política educativa debe asumir este objetivo, desconsiderando la no obtención del éxito y el fracaso como fatalidades imposibles de combatir" (Nóvoa, 2009, p. 90). Dicha preocupación se acerca al énfasis analítico de este trabajo.

Como se señaló anteriormente, se toman como enfoque analítico las concepciones de conocimiento escolar que abarcan la implementación de las políticas de ampliación de la jornada en el contexto de Rio Grande do Sul. Dicho aspecto se hace relevante en la contemporaneidad a medida que, como sugieren Gabriel y Ferreira (2012), "las luchas por la democratización de la escuela pública pasan, en gran medida, por cuestión del conocimiento" (p. 228). En base a esto, aunque los debates sobre el conocimiento escolar se producen en un campo controversial, las autoras proponen que continuemos utilizando este concepto, colocándolo "bajo tachón". Dicha actitud interpretativa, inspirada en los escritos de Stuart Hall, implica "mirar por detrás de las líneas cruzadas que marcan los tachones, desplazando significados previamente fijados y permitiendo la emergencia de 'nuevos' sentidos" (Gabriel y Ferreira, 2012, p. 228).

La opción de investigar el conocimiento escolar, en las condiciones de nuestro tiempo, parte del presupuesto de que conceptos como este "aún son 'buenos para pensar' políticamente el campo académico y la democratización de la escuela pública" (Gabriel y Ferreira, 2012, p. 234). Tal posicionamiento teórico nos permite buscar otros abordajes teóricos pero, al mismo tiempo, pone al conocimiento escolar como un "objeto no posible de contornar" para el análisis crítico de las políticas del programa escolar (Gabriel y Castro, 2013). O aun, en la perspectiva de Michael Young (2010), es importante reiterar que "la adquisición del conocimiento es el propósito-clave que distingue la educación (sea esta básica, pos obligatoria, vocacional o superior) de todas las otras actividades" (p. 174). 
En fin, en este texto, se pretende argumentar que ambas políticas curriculares examinadas atribuyen centralidad a la constitución de "comunidades de aprendizaje". Entienden la ampliación de la jornada escolar como una apuesta a actividades pedagógicas innovadoras, con enfoque en los intereses y las experiencias culturales de los estudiantes. Se diferencian, no obstante, en el modo de organización curricular, a medida que el programa Más Educación se centra en habilidades genéricas, mientras que la Escuela de Turno completo dispone de expectativas de aprendizaje para las diferentes franjas etarias. De acuerdo con los proyectos, se ha percibido una fragilidad de los procesos de selección y organización del conocimiento escolar, visto que dichas políticas curriculares descuidan las posibilidades de transmisión cultural atinentes a la educación escolarizada.

\section{AMPLIACIÓN DE LA JORNADA ESCOLAR: UN CUADRO ANALÍTICO}

Al examinar atentamente las políticas contemporáneas de escolarización, en el contexto nacional e internacional de la actualidad, se percibe una tendencia en el incentivo de prácticas de ampliación de la jornada escolar (Cavaliere, 2007). Generalmente, dichas políticas posicionan la ampliación del tiempo escolar en una perspectiva de resignificación de las culturas escolares, tanto respecto a una multiplicación de programas de protección social, como en lo que se refiere a desplazamientos en el sentido de calidad pedagógica de los procesos formativos en la Educación Básica. Desde el punto de vista teórico, se destcaca que esa problemática atraviesa la producción analítica de diferentes teorizaciones, bien como de contextos múltiples y diferenciados (Coelho, 2009; Moll, 2012).

En el contexto brasileño, aunque la emergencia de la temática transcurre en los movimientos de renovación pedagógica de la primera mitad del siglo XX (Teixeira, 1978), en las últimas dos décadas se ha notado un movimiento de consolidación de la temática de ampliación del tiempo escolar (Cavaliere, 2007). Este movimiento se ha visibilizado tanto en la literatura contemporánea, como en el ordenamiento curricular de las escuelas brasileñas. Dicha consolidación, de forma general, se produce bajo las condiciones de atención a los delineamientos históricos de la educación brasileña, sobre todo respecto al artículo 34 de la Ley $\mathrm{N}$. 9 9.394/96, que versa sobre la posibilidad de ampliación de los tiempos escolares en la Educación Básica. Actualmente, con la multiplicación de nuevos programas gubernamentales, se han intensificado iniciativas en esta dirección, sobre todo con acciones multisectoriales como los programas Más Educación, Segundo Tiempo o Enseñanza Media Innovadora, entre otros. Reforzando ese escenario, todavía necesitamos destacar que el nuevo Plan Nacional de Educación (PNE) encamina en su meta 6 a la ampliación de la jornada escolar en un 50\% de las escuelas brasileñas. En fin, no parece que esta posibilidad de ampliación haya estado en el orden del día de las políticas de escolarización brasileñas de nuestro tiempo. 
En consonancia con ese significativo crecimiento, se ha notado que solamente el programa Más Educación -iniciativa más destacada de las últimas décadas- ha aumentado su amplitud para casi cincuenta mil escuelas en el último año (Figura 1).

Figura 1. Escuelas atendidas por el Programa Más Educación

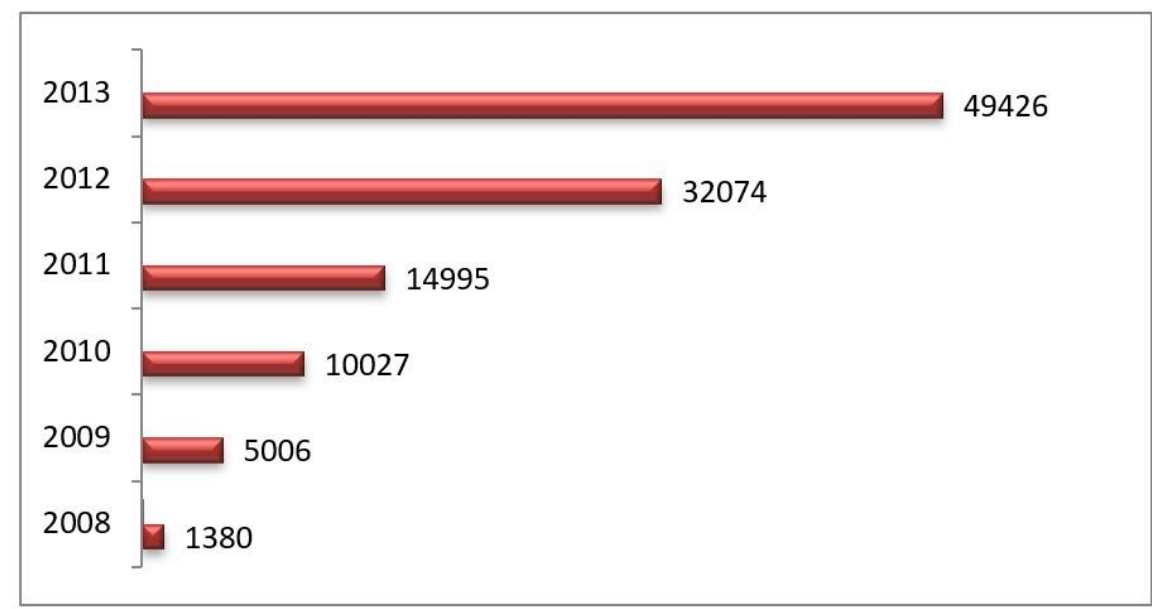

Fuente: Ministério da Educação (2014)

En esa dirección, se hace posible percibir que diferentes conjuntos argumentativos han sido producidos por la sociedad brasileña, proponiéndose justificar la implementación de estas nuevas políticas educacionales. En general, dichos abordajes argumentan acerca de una resignificación de las configuraciones culturales de las familias brasileñas, por los nuevos condicionantes sociales derivados de la ocupación del mercado laboral por las mujeres y también por las restricciones normativas al trabajo infantil, los controles políticos de las nuevas demandas colectivas, vinculadas al riesgo o la vulnerabilidad social, así también como por la mejoría de las condiciones de calidad de las escuelas públicas brasileñas y la consecuente mejoría del desempeño de los estudiantes en las evaluaciones de larga escala (Cavaliere, 2007).

Así, de diferentes modos, la ampliación de la jornada escolar en Brasil ha pasado por los distintos programas políticos de la última década, engendrando un conjunto de nuevos sistemas de raciocinio pedagógico, que tienden a regular las prácticas escolares del país. Vale explicar también que, de acuerdo con Cavaliere (2007), podemos entender por "jornada escolar ampliada" los casos en que la carga horaria sobrepasa a las cuatro horas diarias, incluso en algunos días de la semana.

Específicamente en Rio Grande do Sul, a principios de la década de 1990, se observó la emergencia de un conjunto de iniciativas de creación de escuelas de turno completo. Bajo la gestión del político laboralista Alceu Collares (1991-1995), se desarrollaron en el Estado, noventa y cuatro (94) Centros Integrados de Educación Pública (CIEPs), bajo inspiración de los modelos producidos en Rio de Janeiro en la gestión de Leonel Brizola (y Darcy Ribeiro). Los centros referidos eran concebidos como 
estrategias políticas de educación de turno completo, espacios en los que los sujetos escolares permanecerían en dos turnos escolares, recibiendo aspectos tanto de la educación formal, como se nombraba en la época, como de la educación informal, que correspondería a los demás cuidados pedagógicos como la alimentación, atención odontológica, proyectos comunitarios, formación complementaria, entre otros.

El desafío puesto en esa gramática política era construir escuelas de calidad en barrios pobres, objetivando de acuerdo al discurso del gobernador Alceu Collares, "concientizar que la escuela de turno completo es esencial para la educación de calidad. Sin ella, jamás conseguiremos competir con los demás países" (Partido democrático trabalhista, 2010). Es necesario entender que, en aquel momento, la mejor forma de educar a los niños de las comunidades populares era ampliar su tiempo de permanencia en la escuela, perspectiva que colaboraría tanto para la formación intelectual de tales sujetos, como para el desarrollo del país. Tal argumento se inspiraba en el diagnóstico producido por Darcy Ribeiro, en la segunda mitad de la década de 1980, en el que indicaba la incapacidad de las instituciones brasileñas para la educación de su población (Velloso Maurício, 2004). En esa dirección, les tocaría a las escuelas considerar como principio orientador "el respeto al universo cultural del alumno en el proceso de introducción al dominio del código culto. La escuela debería servir de puente entre la cultura del alumno, que sabe muchas cosas para garantizar su sobrevivencia, y el conocimiento formal exigido por la sociedad" (Velloso Maurício, 2004, p. 41).

Aunque la pauta de dicha política de escolarización sea amplia y diversificada, fueron innumerables las críticas surgidas sobre ese abordaje. Bomeny (2007) sugiere que el programa de los CIEPs en Rio de Janeiro produjo innumerables discontinuidades en su implementación, tanto respecto al modelo de liderazgo populista y personalista de Leonel Brizola, como por la pauta mesiánica del proyecto que presumía un "salvar a través de la escuela". Cavaliere (2007) reconoce los CIEPs como la experiencia más duradera en la educación de turno completo en la historia reciente de Brasil. Entre tanto, describe que, en las experiencias de la ciudad de Rio de Janeiro, puede observarse un efecto paradojal del tiempo, una vez que "la ocupación poco interesante del tiempo completo llevó a la creación de un concepto negativo sobre estas escuelas y a su consecuente vaciamiento" (Cavaliere, 2007, p. 1019). Por otro lado, examinando específicamente una experiencia del Centro Integrado de Educación Pública, desarrollada en el interior de Rio Grande do Sul, Castro, Souza, Werle y Morais (2011) comentan dimensiones exitosas de la calidad pedagógica del trabajo desarrollado en esta institución escolar, sobre todo por las relaciones políticas desarrolladas, descritas como "una escuela en que la participación hace la diferencia".

Para examinar la cuestión en la actualidad, desde su agenda múltiple y heterogénea, analizaré dos conjuntos de prácticas curriculares que se proponen al implementar la ampliación de la jornada escolar. Atribuiré atención al Programa Más Educación, desarrollado en mi contexto investigativo desde el año 2007, y, al mismo tiempo, revisaré el proyecto Escuela de Tiempo Integral, recientemente implementado 
en el estado de Rio Grande do Sul. Este abordaje analítico se distancia de la posibilidad de establecer cuadros interpretativos basados en la comparación; sin embargo, ante esto, deseo producir un breve diagnóstico acerca del espacio ocupado por el conocimiento escolar en las referidas políticas.

\section{BREVE CONTEXTUALIZACIÓN DEL PROGRAMA MÁS EDUCACIÓN}

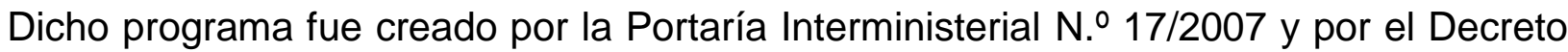

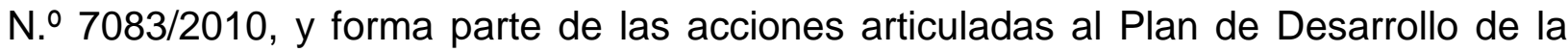
Educación (PDE). Tiene como objetivo inducir a los diferentes sistemas de enseñanza, a través del apoyo técnico y financiero, hacia la ampliación de la jornada escolar en la perspectiva de la educación de tiempo completo. Se organiza en una perspectiva intersectorial, pues involucra diferentes ministerios en la búsqueda de la disminución de las desigualdades educacionales y la valoración de la diversidad cultural de Brasil.

Como una estrategia inductora, el programa Más Educación sugiere que la ampliación de la jornada escolar implica un "alargamiento de la visión sobre la institución escolar" (Ministério da Educação, 2009a, p. 28). Dicho alargamiento produce implicaciones en la relación entre los tiempos, los espacios y los saberes a enseñarse en las escuelas. La búsqueda de diálogo con las comunidades escolares, la composición de acciones integradas o incluso una reaproximación entre escuela y vida, se presentan como presupuestos de acción pedagógica de dicho programa. De forma general, la ampliación de la jornada escolar se posiciona como una estrategia para que las escuelas repiensen sus prácticas y procedimientos, sobre todo al reconocer que todos los espacios son educativos.

Desde el punto de vista curricular, se observa que hay una tendencia a privilegiar un diálogo entre los saberes escolares y los saberes comunitarios, ampliando la articulación entre escuela y comunidad.

La escuela desempeña un rol fundamental en el proceso de construcción y de difusión del conocimiento. Está situada como lugar de diálogo entre los diferentes saberes, las experiencias comunitarias y los saberes sistematizados históricamente por la sociedad en campos de conocimiento y, en esa posición, puede elaborar nuevos abordajes y seleccionar contenidos. Así, el desarrollo integral de los estudiantes no puede ser considerado como responsabilidad exclusiva de las escuelas, sino también de sus comunidades, ya que únicamente juntas pueden resignificar sus prácticas y saberes. De esta forma, la institución escolar es desafiada a reconocer los saberes de la comunidad, además de aquellos trabajados en sus programas, y con ellos promover una constante y fértil transformación, tanto de los contenidos escolares como de la vida social (Ministério da Educação, 2009a, p. 34). 
La articulación entre saberes escolares y saberes comunitarios se presenta en el documento Red de Saberes (Ministério da Educação, 2009b). De acuerdo con el documento antes mencionado, se entienden por saberes comunitarios los aprendizajes producidos desde las experiencias culturales de los estudiantes. Dichos saberes representan "el universo cultural local, es decir, todo aquello que nuestros alumnos traen para la escuela, independientemente de sus condiciones sociales" (p. 37). Para la composición de los saberes comunitarios se indican once áreas a priorizarse (Cuadro 1).

Cuadro 1 - Saberes comunitarios

\begin{tabular}{l}
\multicolumn{1}{c}{$\begin{array}{c}\text { Áreas para la organización } \\
\text { de los saberes comunitarios }\end{array}$} \\
\hline Habitación \\
Cuerpo/vestuario \\
Alimentación \\
Juegos/Juguetes \\
Organización política \\
Condiciones ambientales \\
Mundo del trabajo \\
Curas y rezos \\
Expresiones artísticas \\
Narrativas locales \\
Calendario local \\
\hline
\end{tabular}

Fuente: Ministério da Educação (2009b)

Dichos saberes, conforme indica el documento, tienen que estar articulados a los saberes escolares. La investigación se posiciona como estrategia privilegiada para la movilización de los saberes, considerando que su objetivo es "dinamizar la formación del estudiante a través de un proceso de educación capaz de hacer de la escuela una comunidad de aprendizaje" (Ministério da Educação, 2009b, p. 43). La cuestión de los contenidos, desde ese abordaje, se indica por "enunciados más o menos generales, buscando vincular concepto, aplicación y movilización del conocimiento" (p. 43). En otras palabras, se nota una perspectiva que posiciona los saberes escolares entre el pensar y el hacer. Además de las áreas del conocimiento, hay un énfasis en habilidades, procedimientos y prácticas. 
La articulación entre los saberes se efectiviza a través de determinados macro campos, seleccionados por la comunidad escolar desde una lista previa producida por la gestión nacional del programa. Con respecto a los saberes escolares, enfoque de este estudio, se atribuye atención al modo en que tales conocimientos se diseñan.

Bajo el formato de un mandala (Figura 2), los saberes escolares se presentan en una lista de habilidades: clasificar, sistematizar, debatir, comparar, rever, entre otros. No se han encontrado especificaciones sobre las concepciones epistemológicas o criterios pedagógicos que orientarían las elecciones sobre los contenidos a enseñarse.

Figura 2. Mandala de los Saberes Escolares (Programa Mais Educação)
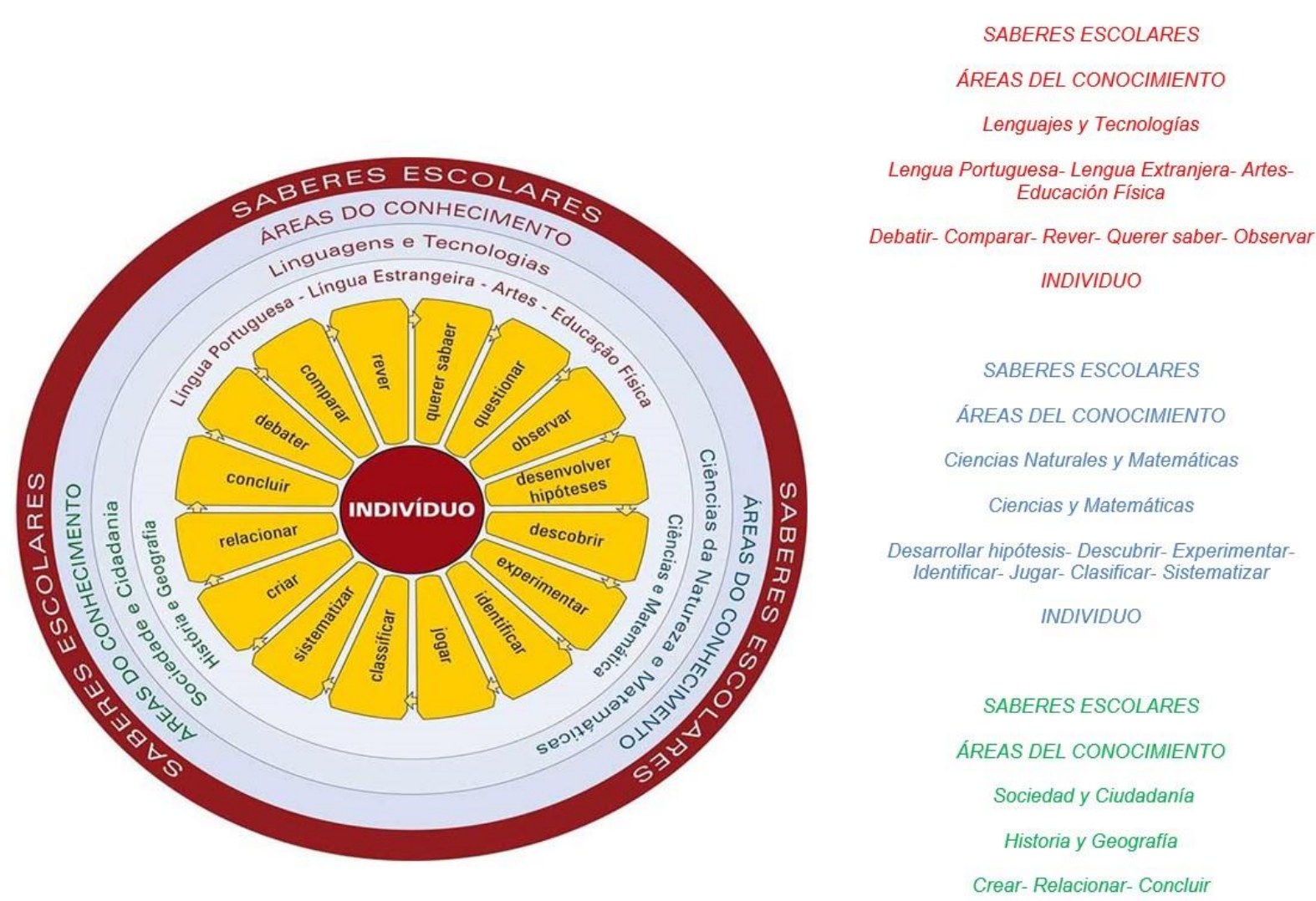

INDIVIDUO

Fuente: Ministério da Educação (2009b)

Respecto al proceso de implementación del programa Más Educación, en el contexto ahora examinado, se nota que hay una predominancia en la articulación entre saberes comunitarios y escolares, priorizando la composición de "comunidades de aprendizajes". 
Los saberes escolares se indican de forma genérica, entre "habilidades, prácticas y procedimientos". De forma general, puede subrayarse provisoriamente que, al distribuir sus actividades en macro campos y talleres, el programa Más Educación es privilegiado de una organización curricular que pone en segundo plano las dimensiones políticas y epistemológicas del conocimiento escolar, así también como los procesos de transmisión cultural. De acuerdo con Young (2007), es importante resignificar la noción de transmisión, atribuyéndole "un significado diferente, que presupone explícitamente el envolvimiento activo del aprendiz en el proceso de adquisición del conocimiento" ( $p$. 1293). A continuación, se examinará la otra política de ampliación de la jornada escolar, en proceso de implementación en Rio Grande do Sul.

\section{ESCUELA DE TIEMPO COMPLETO: ÉNFASIS Y PERSPECTIVAS}

Con enfoque en la Ley del Estado N.⒕461, Rio Grande do Sul presentó una propuesta de reorientación curricular de las escuelas de turno completo, asumiéndola como un desafío para la planificación y la organización de las actividades escolares. Parte del presupuesto, en su argumentación, de que no basta ampliar el tiempo de permanencia de los estudiantes en la Enseñanza Fundamental, "sino de reestructurar las bases de tiempo/aprendizaje, privilegiando una formación humanista y de inclusión social" (Secretaria da Educação do Rio Grande do Sul, 2014, p. 3). El proyecto Escuela de Tiempo Completo, en su propuesta pedagógica, busca "la construcción del aprendizaje de los estudiantes en una jornada escolar ampliada que oferte actividades escolares educativas y diversificadas de forma articulada" (p. 4).

Desde el punto de vista teórico, la propuesta se inspira en el "progresismo pedagógico", indicando que la posibilidad de formar sujetos integralmente es un ideal formativo desde la Paideia griega. Subraya, no obstante, que será en la Modernidad que la búsqueda por la emancipación humana destinará la escuela pública para este fin. Reconociendo la experiencia desarrollada en Brasil a lo largo del siglo XX, en la que las conclusiones de Anísio Teixeira y Darcy Ribeiro adquieren destaque, la documentación pedagógica sugiere que el proyecto del Estado no pretende organizarse como "una yuxtaposición de turnos de trabajo" (Secretaria da Educação do Rio Grande do Sul, 2014, p. 7). Será presentada, entonces, una concepción de educación integral posicionada desde un nuevo prisma formativo.

Se propone, así, una escuela de educación integral, que actúe como una comunidad de aprendizaje, en la que los jóvenes desarrollen una cultura democrática, solidaria y participativa, por medio del protagonismo en actividades transformadoras, aprendiendo a ser autónomos al formular y ensayar la concretización de proyectos de vida y de sociedad (Secretaria da Educação do Rio Grande do Sul, 2014, p. 7). 
La escuela de turno completo, desde el proceso de implementación en curso en Rio Grande do Sul, pretende producir una dimensión formativa que sobrepase las posibilidades de una educación escolar basada en la transmisión de conocimientos. Delinea, en esa dirección, la posibilidad de "la efectuación de nuevas actitudes, tanto respecto a la cognición como a la convivencia social, privilegiando los cuatro pilares de la Educación adoptados por la UNESCO" (Secretaria da Educação do Rio Grande do Sul, 2014, p. 11). La formación escolar de los niños y adolescentes del referido contexto, sobre todo aquellos en situación de exclusión, vulnerabilidad social o privaciones, adquiere, en la argumentación desarrollada, mayores posibilidades de garantía de la calidad social.

En esta propuesta pedagógica, la escuela de turno completo se posiciona estratégicamente en la articulación entre calidad de la enseñanza e inclusión social. La ampliación de la carga horaria para ocho horas diarias, asociada a una matriz curricular flexible y la participación de la comunidad escolar, son algunas apuestas evidenciadas en la nueva política. Sobre ese aspecto, el programa escolar se dimensiona desde nuevas bases.

La Escuela de Turno Completo, acompañando al Proyecto Político Pedagógico, presentado como marco para la Red del Estado de Enseñanza, considera al estudiante en sus aspectos biológicos y emocionales, pasando por transformaciones que dialogan con su proceso de aprendizaje, considerado este como su ritmo y especificidades en lo que el conocimiento y el programa escolar se integran y constituyen, estableciendo una amplia red de significaciones, reafirmando la escuela como lugar de construcciones y acciones colectivas. Esto pasa, necesariamente, por la interlocución, la escucha de todos los segmentos de la Comunidad Escolar. El programa es interdisciplinario, rescatando el ser en su unidad y diversidad, en lo que la construcción del conocimiento pasa por el trabajo colectivo, la cultura de paz y el reconocimiento y acogimiento de las diferencias de los estudiantes (Secretaria da Educação do Rio Grande do Sul, 2014, p. 13).

El programa escolar presentado bajo esa orientación pedagógica, se fundamenta desde cuatro fuentes, a saber: epistemológica, filosófica, socio-antropológica y sociopsicopedagógica. Sobre dichas bases, el programa de la Escuela de Tiempo Completo, en el estado de Rio Grande do Sul, busca contemplar "los procesos que se relacionan al lugar y el desarrollo sostenible, valorando los saberes locales y los nuevos conocimientos $y$, de esa manera, rescatando la importancia de la escuela para la comunidad donde se inserta" (Secretaria da Educação do Rio Grande do Sul, 2014, p. 14). Acerca de esta perspectiva, podemos inferir la relevancia de los saberes y las experiencias provenientes de las comunidades locales, considerando como principios orientadores del trabajo pedagógico a los tópicos expresados en el cuadro a continuación (Cuadro 2). 
Cuadro 2 - Principios orientadores del trabajo pedagógico

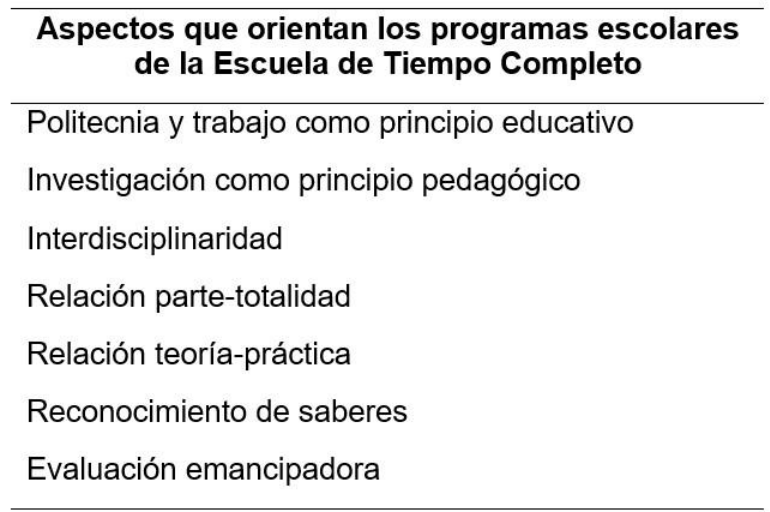

Fuente: Secretaria da Educação do Rio Grande do Sul (2014)

En común con estos principios podemos indicar una preocupación con tres cuestiones específicas. La primera de esas es el desarrollo de actividades escolares adecuadas al contexto y a las demandas comunitarias. Otro aspecto se refiere a la promoción de formas alternativas de integración curricular, que puedan favorecer una articulación entre los diferentes saberes y actividades desarrolladas en la institución escolar. La tercera cuestión derivada de esta propuesta curricular enfatiza la promoción de experiencias formativas que desarrollan los estudiantes integralmente.

Desde el punto de vista de la operación de esta propuesta curricular, la escuela de turno completo, en implementación por el estado de Rio Grande do Sul, se organiza de forma diferente en relación a la política curricular anteriormente descrita. La composición curricular de estas escuelas se ancla en metodologías que buscan atender las fases del desarrollo de cada estudiante; en una matriz curricular flexible, capaz de articular la formación general y las actividades curriculares complementarias; en la organización del espacio en salas ambientadas y en el compromiso con el aprendizaje. El documento examinado, en un ejercicio de proposición de actividades y de saberes a enseñarse, organiza los aprendizajes necesarios para cada franja etaria, clasificados como infancia (6 a 8 años de edad), segunda infancia (9 a 11 años) y adolescencia (12 a 14 años).

En el periodo referente a la infancia, por ejemplo, el documento indica los siguientes aspectos que los aprendizajes deben contemplar (cuadro 3). 
Cuadro 3 - Aprendizajes necesarios para la infancia

Aspectos a contemplarse

* La alfabetización y la literacidad;

* El desarrollo de las diversas formas de expresión, que incluyen el aprendizaje global e interdisciplinario;

* El espacio de apropiación y producción de conocimiento, donde el afecto, la cognición y la ludicidad caminen juntos e integrados;

* Movilidad en las aulas y actividades que lleven a los estudiantes a explorar más intensamente los diversos lenguajes; de la literatura, las artes y a utilizar materiales que ofrezcan condiciones de raciocinar y crear manejando y explorando sus características y propiedades.

Fuente: Secretaria da Educação do Rio Grande do Sul (2014)

La distribución de las actividades se propone integrar la formación general con las actividades curriculares electivas, rompiendo con la lógica del turno y contra turno. Además de las áreas del conocimiento, integran las actividades propuestas para la Escuela de Turno Completo: iniciación a la investigación, lectura y producción textual, experiencias matemáticas, educación en derechos humanos, arte y cultura, deporte y recreación, orientación de estudios, lecturas y centro de lenguas. La articulación entre dichas actividades, en la propuesta pedagógica examinada, toma como objetivo el reconocimiento de la escuela como un "espacio placentero de construcción del conocimiento, espejando la responsabilidad con una educación de calidad social hacia la inclusión" (Secretaria da Educação do Rio Grande do Sul, 2014, p. 26).

En ese proyecto también hemos notado una predominancia de presupuestos pedagógicos destinados a promover espacios de diálogo e interlocución, a manera de potencializar la constitución de "comunidades de aprendizaje". Sin embargo, se diferencia en sus estrategias de intervención, tanto respecto a la promoción de acciones articuladas entre turno y contra turno, como en la búsqueda de la definición de expectativas de aprendizaje para las diferentes franjas etarias. Vale señalar aun el privilegio de modelos formativos basados en el aprendizaje permanente, las demandas comunitarias y la integración curricular.

De forma general, también podemos notar una intensa preocupación con las cuestiones de la protección social de la infancia, evidenciada recurrentemente. Ese contexto analítico se acerca a la conclusión de Fabris y Traversini (2011) sobre la escuela contemporánea, en la que "los conocimientos se van postergando, y el enfoque está en atender a los sujetos en sus daños y perjuicios" (p. 7). A continuación, se finalizará el abordaje para este texto, recapitulando algunas balizas teóricas que han orientado los presentes estudios sobre el programa y el conocimiento escolar. 


\section{POLÍTICAS CURRICULARES Y AMPLIACIÓN DE LA JORNADA ESCOLAR: ALGUNOS AMARRES}

Aunque la ampliación de la jornada escolar se considera un importante movimiento hacia la democratización de la escolarización de Brasil, en las referidas políticas curriculares se nota un excesivo énfasis en las prácticas y los intereses de las comunidades en que los estudiantes se insertan. Desde el punto de vista teórico, este trabajo se asocia a los indicativos de Young (2013) en los que "los programas educacionales y, consecuentemente, los estudios del programa deben partir no del alumno como aprendiz, sino del derecho del alumno o de su acceso al conocimiento" (p. 18). De esta forma, los Estudios Curriculares, campo en el que se inscribe este estudio, tienen el compromiso de "analizar y criticar los programas existentes y explorar las diferentes formas que ellos pueden tomar" (p. 18). Las políticas examinadas priorizan la constitución de comunidades de aprendizaje, ahora centradas en habilidades genéricas, y definidas por la construcción de expectativas de aprendizaje para las etapas del desarrollo humano. Sin entrar en las dimensiones políticas de este principio de los aprendizajes permanentes (Lima, 2012; Popkewitz, 2009), interesa enfocar la cuestión del conocimiento escolar, en las formas por las que se sitúa en las políticas analizadas.

Cabe aclarar que en este trabajo se hizo uso de una concepción de conocimiento escolar que reconoce su relevancia social y política para la democratización de la educación pública ${ }^{4}$. Como sugieren Gabriel y Ferreira (2012), aunque situado en un campo controversial, el conocimiento escolar todavía sirve para pensar la escolarización, siempre que sea situado en otras articulaciones. Juntamente con Moreira (2013), se entiende que la institución escolar, "además de enseñar conocimientos que expliquen mejor el mundo (lo que seguramente necesita hacer), busque subsidiar y comprometer al alumno hacia esfuerzos por el cambio de este mundo" (p. 41). El conocimiento en la escuela, desde esta concepción, se presenta como una cuestión curricular central, tanto de orden epistemológico como de justicia social. El abordaje de Young (2013) permite aproximar la objetividad del conocimiento a enseñarse en las escuelas.

Yo supongo que la cuestión del programa educacional “¿qué conocimiento?”, es tanto una cuestión epistemológica que define lo que debe constituir el derecho de los estudiantes en etapas y en áreas de especialización diferentes, como una cuestión de justicia social sobre el derecho al conocimiento por parte de todos los alumnos, sin tener en cuenta si el conocimiento es rechazado o considerado difícil. Si algún conocimiento es "mejor", ¿cómo podemos negarlo a todos los alumnos y permitir que algunos, como hacemos en Inglaterra, sean limitados al "conocimiento sin poder" a partir de la edad de 14 o 16 ? (p. 20).

\footnotetext{
${ }^{4}$ Recientemente hemos direccionado nuestros estudios hacia la cuestión del conocimiento escolar (Silva y Pereira, 2013; Silva, 2014; Silva, Vial Ribeiro, Cigognini, y Brancer Fávero (2014).
} 
Retomar el conocimiento escolar, en el debate curricular actual, trae implicaciones significativas para las prácticas de ampliación de la jornada. Como recuerdan Gabriel y Cavaliere (2012), aunque las formas escolares están en crisis, "no significa que hayan sido condenadas y vaciadas de cualquier posibilidad de subversión" (p. 292). Las ponderaciones acerca del programa de la educación integral, todavía conforme las autoras, comprenderían "el programa escolar no como algo inmutable, y reconocer que los contenidos no son objetos estables y universales, ni tampoco una adaptación didáctica del conocimiento científico, sino construcciones específicas, tanto desde el punto de vista político como epistemológico" (p. 292).

En ese camino, al dirigir las preocupaciones para las políticas de ampliación de la jornada escolar en implementación en Rio Grande do Sul, se optó por estrechar los vínculos con una concepción centrada en la relevancia política y epistemológica del conocimiento escolar en los programas, vislumbrando sus potencialidades en la ampliación del repertorio cultural de los estudiantes. Inclusive, como provoca el epígrafe de este texto, junto a António Nóvoa (2009), ¿qué más esperamos de la educación además de sobrepasar las fronteras trazadas como destino?

\section{REFERENCIAS}

Bomeny, H. (2007). Salvar pela escola: programa especial de educação. Sociologia, Problemas e Práticas, 55, 41-67. Recuperado de http://www.scielo.mec.pt/pdf/spp/n55/n55a04.pdf

Castro, M., Souza, M., Werle, F., y Morais, V. (2011). Escola Estadual Neusa Mari Pacheco: a School Where Participation Makes a Difference. Journal of the International Society for Teacher Education, $15,76-81$.

Cavaliere, A. (2007). Tempo de escola e qualidade na educação pública. Educação e Sociedade, 28(100), 1015-1035.

Cavaliere, A. M., y Velloso Maurício, L. (2012). A ampliação da jornada escolar nas regiões Nordeste e Sudeste: sobre modelos e realidades. Revista Educação Em Questão, 42(28). Recuperado de https://periodicos.ufrn.br/educacaoemquestao/article/view/4060

Coelho, L. (2009). História(s) da educação integral. Aberto, 22(80), 83-96.

Dubet, F. (2011). Mutações cruzadas: a cidadania e a escola. Revista Brasileira de Educação, 16(47). https://doi.org/10.1590/S1413-24782011000200002

Fabris, E., y Traversini, C. (2011, octubre). Conhecimentos escolares sob outras configurações: efeitos das movimentações disciplinares e de controle. Presentado en la 34ํㅜ Reunião Anual da ANPED, Rio de Janeiro, Brasil.

Gabriel, C., y Ferreira, M. (2012). Disciplina escolar e conhecimento escolar: conceitos sob rasura no debate curricular contemporâneo. En J. Libâneo y N. Alves (Orgs.), Temas de pedagogia: diálogos entre didática e currículo (pp. 227-241). São Paulo, Brasil: Cortez. 
Gabriel, C., y Cavaliere, A. (2012). Educação integral e currículo integrado: quando dois conceitos se articulam em um programa. En J. Moll (Comp.), Caminhos da educação integral no Brasil: direito a outros tempos e espaços educativos (pp. 277-294). Porto Alegre, Brasil: Penso.

Gabriel, C., y Castro, M. (2013). Conhecimento escolar: objeto incontornável da agenda política educacional contemporânea. Educação em Questão, 45(31), 82-110.

Libâneo, J. (2012). O dualismo perverso na escola pública brasileira: escola do conhecimento para os ricos, escola do acolhimento social para os pobres. Educação e Pesquisa, 38(1), 13-28.

Lima, L. (2012). Aprender para ganhar, conhecer para competir: sobre a subordinação da educação na "sociedade da aprendizagem". São Paulo, Brasil: Cortez.

Ministério da Educação. (2009a). Educação Integral: texto de referencia para el debate nacional. Recuperado de http://portal.mec.gov.br/dmdocuments/cadfinal_educ_integral.pdf

Ministério da Educação. (2009b). Rede de Saberes - Mais Educação: presupuestos para proyectos pedagógicos de educación integral. Recuperado de http://portal.mec.gov.br/dmdocuments/cad_mais_educacao_2.pdf

Ministério da Educação. (2014). Programa Mais Educação. Recuperado de http://portal.mec.gov.br/index.php?option=com_content\&view=article\&id=16690\&ltemid=1115.

Moll, J. (2012). A agenda da educação integral: compromissos para sua consolidação como política pública. En J. Moll (Comp.), Caminhos da educação integral no Brasil: direito a outros tempos e espaços educativos (pp. 129-146). Porto Alegre, Brasil: Penso.

Moreira, A. (2013). A importância do conhecimento no currículo e os pontos de vista de Michael Young. En M. Favacho, J. Pacheco, y S. Sales (Comps.), Currículo, conhecimento e avaliação: divergências e tensões (pp. 33-47). Curitiba, Brasil: CRV.

Nóvoa, A. (2009). Professores: imagens do futuro presente. Lisboa, Portugal: Educa.

Popkewitz, T. (2009). El cosmopolitismo y la era de la reforma escolar. Madrid, España: Morata.

Partido democrático trabalhista. (2010). Partido democrático trabalhista: nossas bandeiras. Recuperado de http://www.pdt.org.br/index.php/nossas-bandeiras/educacao/mais-sobre-os-cieps/os-cieps-dorio-grande-do-sul.

Secretaria da Educação do Rio Grande do Sul. (2014). Documento orientador para a reestruturação curricular das escolas em tempo integral - enseñanza fundamental. Recuperado de http://servicos.educacao.rs.gov.br/dados/dp_cga_doc_orient_tempo_integ.pdf

Silva, R. R. D. (2014). Políticas de constituição do conhecimento escolar para o Ensino Médio no Rio Grande do Sul: uma analítica de currículo. Educação em Revista, 30(1), 127-158.

Silva, R. R. D., y Pereira, A. (2013). Políticas de constituição do conhecimento escolar na pesquisa educacional brasileira. Cadernos de Pesquisa, 43(150), 884-905.

Silva, R. R. D., Vial Ribeiro, C. R., Cigognini, F. C., y Brancer Fávero, S. (2014). O conhecimento escolar nas políticas de ampliação da jornada escolar. Saberes e fazeres educativos, 13(1), 22-26.

Teixeira, A. (1978). Pequena introdução à filosofia da educação. São Paulo, Brasil: Editora Nacional. 
Velloso Maurício, L. (2004). Literatura e representações da escola pública de horário integral. Revista Brasileira de Educação, 27, 40-56.

Young, M. (2007). Para que servem as escolas?. Educação e Sociedade, 28(101), 1287-1302.

Young, M. (2010). Conhecimento e currículo: do socioconstrutivismo ao realismo social na sociologia da educação. Porto, Portugal: Porto Editora.

Young, M. (2013). A superação da crise em estudos curriculares: uma abordagem baseada no conhecimento. En M. Favacho, J. Pacheco, y S. Sales (Comps.), Currículo, conhecimento e avaliação: divergências e tensões (pp. 11-31). Curitiba, Brasil: CRV. 